\title{
Physical modelling of electroporation in close cell to cell proximity environments
}

P. T. Gaynor ${ }^{1}, \&$ P. S. Bodger ${ }^{1}$.

${ }^{1}$ Department of Electrical and Computer Engineering.

University of Canterbury

Private Bag 4800

Christchurch, New Zealand.

Corresponding Author: Paul Gaynor

Ph: ++6433642269

Fax: ++6433642761

Email: paul.gaynor@canterbury.ac.nz

Short Title: Physical Modelling of Electroporation 


\section{ABSTRACT}

Many applications of electroporation, especially those utilizing electrofusion and in-vivo electroporation, involve cell environments that include close cell to cell proximity and a wide range of target cell size. It is important to understand how this kind of environment may alter optimum electroporation electrical parameters for any given application. A physical, electrically equivalent model of biological cell electroporation, based on aqueous solution filled thin latex rubber membrane spheroids, was used to investigate membrane permeabilization behaviour where there is both close cell to cell proximity and different cell radii.

Cell model arrangements were pulsed using either a $50 \mu \mathrm{s}$ or $10 \mu \mathrm{s}, 1 / e$ decay time constant DC capacitive discharge electric field, with peak amplitudes of $160-500 \mathrm{kVm}^{-1}$.

Results indicate that compared to cells in isolation, electroporation initiates at substantially decreased applied electric field magnitudes in regions of close cell to cell proximity where the external media conductivity is lower than the cell interior conductivity, and the membrane is maximally polarized. Additionally, the use of shorter time constant, higher peak magnitude pulse parameters should reduce the relative difference in threshold membrane permeabilization in regions of close cell to cell proximity for cells of different size so that the degree of electroporation is more uniform for variable size and shape target cell populations.

PACS Classification numbers: 87.16.Ac, 87.17.Aa, 87.50.Rr, 77.22.Jp, 52.80.Wq, 84.70.+p, and 07.50.-e. 
KEYWORDS: electroporation, electropermeabilization, physical modelling, in-vivo, pulsed. 


\section{INTRODUCTION}

Biological electroporation (or electropermeabilization) is the process of electric field-induced poration (permeabilization) of the phospho-lipid bilayer membrane that encompasses cell contents (Neumann et al., 1999; Weaver and Chizmadzhev, 1996). Electroporation is a very useful biophysical effect that has been utilized in many applications.

Numerous current and new applications of electroporation involve environments where target cells may be in close cell to cell proximity and of substantially different size and/or shape. For example, electroporation in electrochemotherapy (Hofmann et al., 1999; Singh and Dwivedi, 1999; Serša et al., 2000), electro-gene therapy (Yamazaki et al., 2000; Goto et al., 2000), transdermal drug delivery (Misra et al., 2000; Zewert et al., 1999), and electrofusion in mammalian cloning (Gaynor et al., 2005; Oback et al., 2003; Tatham et al., 1996), all include close cellular proximity and cells of different sizes that should all be simultaneously and optimally electropermeabilized.

It is the desired optimal electropermeabilization of all target cells that necessitates a good understanding of how close cellular proximity and variable cell size affects critical factors such as threshold membrane electropermeabilization, and optimum pulse parameters.

Empirical biological experimentation can yield satisfactory results for a given application but often does not provide significant insight to probable optimal parameters in substantially different applications. Such insight requires qualitative data. Owing to the microscopic scale involved in biological systems, obtaining qualitative information is often difficult to achieve (especially for in-vivo applications). Additionally, biological experimentation can be resource intensive, requiring substantial time, technical skills, and funding.

The usual alternative to actual biological experimentation is mathematical/numerical modelling. These models produce the greatest volume of simulation data and do so with the minimum of resources. Indeed, recent numerical modelling has yielded new insight into aspects of 
electropermeabilization for both isolated and tissue-type structures (Gowrishankar and Weaver, 2003; Jayaram and Boggs, 2004; Tarek, 2005; Ramos et al., 2003; Pavlin et al., 2002; Kotnik and Miklavčič, 2000; Brandisky and Daskalov, 1999). However, mathematical models are always approximations and it is often difficult to accurately model all the non-linear behaviour that may exist in the real system.

Physical modelling offers an alternative to numerical modelling and full biological experimentation. An appropriate physical model can simulate predetermined important biological conditions, and is easily setup, measured and adjusted. Physical models by their nature are able to yield accurate data that would have been extremely difficult to achieve through mathematical (numerical) means owing to possible non-linear responses to system variables, and due to factors that may not have been anticipated in advance.

A previously developed electrically equivalent physical model of biological cells, based on aqueous solution filled thin latex rubber membrane spheroids - known as the 'balloon' model (Gaynor and Bodger, 1994, 1995b), has been adapted to simulate close cell to cell proximity with different cell sizes in order to investigate their effects on membrane permeabilization behaviour. While this model is substantially electrically equivalent up to initiation of biological cell membrane permeabilization, the molecular and physical dynamics of pore formation between the model and cells are very different. The model membrane undergoes dielectric breakdown, resulting in ionization of the membrane material that creates microscopic-scale transmembrane pores. This pore formation is irreversible owing to the non-fluidic mechanical properties of latex rubber membranes. Conversely, it is extensively accepted that cell membranes experience electrically induced non-ionizing molecular rearrangement that creates nanoscopic-scale electropores (Neumann et al., 1999; Weaver and Chizmadzhev, 1996). This pore formation is fluidic, owing to the fluidic nature of the phospho-lipid bilayer, and induced pores may decrease in size and disappear, decrease to a stable size, or increase in size which may lead to cell lysis (Smith et al., 2004). Additionally, owing to the scaling of the model, 
characteristic relaxation time constants are much slower than for biological cells. For example, the model spheroid membrane relaxation time constant is around $10^{-5} \mathrm{~s}$, whereas biological cell membrane relaxation times typically range around $10^{-7}-10^{-6} \mathrm{~s}$. The slower charge separation time constants in the model reinforce the fact that the fundamental mechanisms of membrane permeabilization are different between the model and biological cells. Owing to the fundamental differences in pore dynamics once permeabilization has been initiated, the model is best suited, at this stage, for determination of electric field pulse characteristics leading to onset (or threshold) of electroporation.

\section{ELECTROPORATION THEORY}

Primarily non-conducting membranes bounded on both surfaces by relatively conducting media experience significant electrical polarization upon application of an electric field (Jones, 1995). This membrane polarization creates a transmembrane potential. If the transmembrane potential becomes too large, the membrane will experience an electric field strong enough to induce breakdown (either ionizing or non-ionizing mechanisms depending on the membrane physical properties) at specific points in the membrane. The result is permeabilization, or poration, of the membrane (hence electroporation).

Biological electroporation can occur as cell membranes are relatively non-conducting and are usually bounded externally and internally by relatively conducting media (Neumann et al., 1989; Weaver and Chizmadzhev, 1996). Areas of cell membranes that undergo electroporation occur in regions where polarization is maximized. Along with other factors, maximum polarization is a function of both electric field amplitude and cell geometry. Where the electric field amplitude and cell dimension are

jointly greatest, the membrane polarization is maximized. To illustrate this situation, typical subelectroporation induced transmembrane polarization magnitude (that is, transmembrane potential) distribution of a spherical cell with radius $a$, exposed to a uniform electric field is depicted in Figure 1. While offering no qualitative information, Figure 1 helps explain the rationale behind the required model spheroids orientation between the electrodes, such that maximal transmembrane potential is created in the region of close spheroid-spheroid proximity. Assuming the membrane conductivity $\sigma_{m}$, 
is much less then either the surrounding or interior media conductivities ( $\sigma_{e}$ and $\sigma_{i}$ respectively), then the induced transmembrane potential $U_{m}(t)$, prior to breakdown, from an arbitrary uniform electric field pulse $E(t)$, can be approximated by (Holzapfel, et al., 1982; Gaynor and Bodger, 1995a)

$$
U_{m}(t)=\frac{-1.5}{\tau_{r}} a \int_{0}^{t_{p}} E(t) e^{-\left(\frac{t_{p}-t}{\tau_{r}}\right)} d t \cos \phi
$$

Where $\tau_{r}$ is the membrane relaxation time constant, $t_{p}$ is the application time of the pulse, and $\phi$ is the angle around the cell from the axis formed by the line passing through its origin and parallel to the uniform applied electric field. Under the same assumptions, the membrane relaxation time constant can be approximated by (Qin, et al., 2005)

$$
\tau_{r}=a C_{m}\left(\frac{1}{\sigma_{i}}+\frac{1}{2 \sigma_{e}}\right)
$$

Where $C_{m}$ is the specific membrane capacitance, and is often calculated from (Gaynor and Bodger 1995b)

$$
C_{m}=\frac{\varepsilon_{0} \varepsilon_{r}}{d}
$$

Where $\varepsilon_{0}$ is the permittivity of free space, $\varepsilon_{r}$ is the relative permittivity of the membrane material, and $d$ is the membrane thickness. When multiple cells are packed in a lattice, $U_{m}(t)$ is altered owing to the deformation of the applied electric field by the adjacent cells. It can be shown that the steady-state (DC) $U_{m}$ for lattice-array closely-packed cells is approximated by (Qin, et al., 2005)

$$
U_{m}=-1.5\left(\frac{\frac{3}{2+\left(1-f_{o}\right)^{3 / 2}}}{1+\left(\frac{3 f_{o}}{4 N \pi}\right)^{1 / 3}}\right) E_{p} a \cos \phi \quad\left(0.8 \leq f_{o} \leq 1\right)
$$

Where $f_{o}$ is the volume fraction of cells dispersed in the external medium (an $f_{o}=0$ would be equivalent to no cells, and an $f_{o}=1$ would be equivalent to cells being packed such that they were in contact with all neighbouring cells), and $N$ is the number of cells in a unit simple-cubic, body-centredcubic, or face-centred-cubic lattice. 
Substantially accurate transmembrane potentials for sub-breakdown conditions can be calculated for simple cell geometries (Gaynor and Bodger 1995a and b; Qin, et al., 2005; Kotnik and Miklavčič, 2000). However, the dynamic aspects of membrane electroporation and effect of variable cell-system geometry are extremely difficult to model, although a number of models do exist for both single cells and tissue systems (Jayaram and Boggs, 2004; Tarek, 2005; DeBruin and Krassowska 1999, Smith et al., 2004, Gowrishankar and Weaver, 2003, Ramos et al., 2003).

What yet remains to be accurately modelled, and is not included in any of the models referenced above, is the effect of electro-deformation on electroporation dynamics, particularly with respect to cells in close proximity. Electro-deformation is a well documented and partially modelled process for cells in isolation (Riske and Dimova, 2005; Zimmermann et al., 2000; Sukhorukov, et al., 1998). For cellular environments where the external media is of a lower conductivity than the cell interior (cytoplasm), and the electric field pulse rise time is shorter than the membrane relaxation time constant, then cells experience a deformation force parallel to the electric field axis. The magnitude of this force has not yet been well defined. If the conductivity of the external media is greater than the cell cytoplasm, then no elongation along the electric field axis is induced, and can be calculated as (Sukhorukov et al., 1998)

$$
\frac{\Delta l}{l_{0}}=\alpha E^{2}\left(\frac{\sigma_{i}-\sigma_{e}}{\sigma_{i}+2 \sigma_{e}}\right)
$$

Where $l_{0}$ is the original cell dimension (prior to electric field application) parallel to the applied electric field $E, \Delta l$ is the change in cell length (after application of the electric field) parallel to $E, \sigma_{i}$ is the cell interior conductivity, $\sigma_{e}$ is the external media conductivity, and $\alpha$ is the elastic deformation factor. The elastic deformation factor defines the relative ease of deformation where a value of zero indicates a non-deformable state. The value of $\alpha$ is not analytically defined and must be determined experimentally. 
Electro-deformation force appears on the cell with an extremely short time constant relative to the membrane relaxation time constant, such that its effects precede those of electroporation.

The effect of electro-deformation on electroporation dynamics for cells in close proximity, while rationalised (Zimmermann, et al., 2000), has not yet been well defined in any models. It has been observed that electrofusion is substantially improved if conditions exist where cellular elongation parallel to the applied electric field occurs (Zimmermann, et al., 2000), such that the membranes in contact experience significant compressive forces prior and additional to the electro-compressive force experienced during membrane polarization.

\section{MATERIALS and METHODS}

\subsection{Balloon models}

Latex rubber membrane spheroids (Alpen Products Pty Ltd., Archerfield, Queensland, Australia) were filled with domestic water until the desired radius was reached. The conductivity of the domestic water supply was measured with a liquid conductivity meter (CYBERSCAN PC 300, Eutech Instruments, Singapore) to be $14 \mathrm{mSm}^{-1}$. Two manufacturer specific sizes were used so that the filled and expanded spheroid radii were either $65 \mathrm{~mm}$ or $30 \mathrm{~mm}$. Maintaining these radii ensured a consistent average membrane thickness (measured to be $30 \pm 5 \mu \mathrm{m}$ with a standard micrometer). During filling, small non conducting weights were inserted into the spheroids so that when placed into a water environment they would consistently align vertically. The balloon model membrane was both water-tight and hydrophilic.

PVC rod arrangements were constructed to reproducibly position the four balloon model structures tested between the electroporation tank electrodes. 


\subsection{Electroporation tank}

A rectangular polypropylene tank $(0.540 \times 0.340 \times 0.330 \mathrm{~m})$ was filled with partially deionized water

$\left(6.0 \mathrm{mSm}^{-1}\right)$. The water conductivity was decreased to ensure the model membrane relaxation time constant $\tau_{r}$ is in the same order of magnitude as the pulse decay time constants. This measure has created the condition where it is expected that electro-deformation elongation of the spheroid parallel to the applied electric field will occur during pulsing, as the suspension water has a lower conductivity than the spheroid interior. Two stainless steel plate electrodes $(0.340 \times 0.300 \mathrm{~m})$ were attached to opposing inside surfaces of the tank, resulting in an electrode separation of $0.310 \mathrm{~m}$. A polypropylene bar was fixed across the top of the tank for connecting the balloon model positioning rods to.

\subsection{Balloon model structures}

Four balloon model structures were employed with the two specified radii to simulate various close cell to cell proximity situations that are commonly encountered in many electroporation applications. Three of the structures were two spheroid couplets. The couplets were formed from either two $65 \mathrm{~mm}$ spheroids, two $30 \mathrm{~mm}$ spheroids, or one $65 \mathrm{~mm}$ and one $30 \mathrm{~mm}$ spheroid. Positioning of the couplets was primarily with the close proximity region along an axis normal to the electrode surfaces. The fourth structure was formed with twenty seven, $30 \mathrm{~mm}$ spheroids in a basic cubic $3 \times 3 \times 3$ array. These four structures are illustrated individually suspended in the electroporation tank in Figure 2. For each model structure, all individual spheroids were allowed to have their entire surface exposed to the electroporation tank water before final positioning was achieved. Thus, although spheroids had regions of very close proximity, there was always a thin layer of water separating them.

\subsection{DC pulse generator}

Five stages of a 14-stage variable 1.4 MV inverted Marx impulse generator were used to deliver capacitive discharge DC voltage pulses to the electrodes of the electroporation tank. The discharge rate was fixed to the two experimental 1/e decay time constant $\left(\tau_{p}\right)$ pulses of $50 \mu$ s and $10 \mu \mathrm{s}$, with a 
parallel connected water resistor. The water resistor was doped with sodium chloride to reduce its electrical resistance until the desired time constant was reached.

\subsection{Measurement equipment}

Super-threshold dielectric breakdown of the balloon model membrane (that is, electroporation) during pulsing was photographically captured on 3200 ASA black and white film with a manual shutter control camera (Pentax, ME super). However, threshold onset of electroporation was not determined by photographic evidence as it was not sensitive enough to detect that condition. Pulse voltage waveforms were recorded on a storage oscilloscope (Hewlett-Packard, HP54616B) measuring the output of a $600 \mathrm{kV}$ capacitive voltage divider (Ferranti, London, England) connected to the pulse generator. A typical measured pulse waveform of a $65 \mathrm{kVp}, 50 \mu \mathrm{s} 1 / e$ decay time constant is shown in Figure 3.

The complete experimental setup is illustrated in Figure 4.

\subsection{Determination of electroporation}

In order to normalize the degree of membrane breakdown, results have been determined at the threshold of electroporation. Threshold electroporation has been defined in this study as the observation of between $2-4$ seeping water droplets on the spheroid membrane surface after removal from the electroporation tank, indicating the presence of transmembrane pores induced by the electric field pulse (electropores). The presence of electropores was confirmed through observation by scanning electron microscope. Expanded membrane tension was maintained for electron microscope analysis by adhering $10 \mathrm{~mm}$ interior diameter steel rings to the outer membrane surface encircling the

electropores. The electric field peak, $\mathrm{E}_{\mathrm{p}}\left(\mathrm{Vm}^{-1}\right)$, was calculated by dividing the measured peak voltage by the electrode separation distance.

Whilst the threshold of electroporation does not complete the entire electroporation process (pore density and size increase, and in the case of biological membranes, pore resealing), it does indicate the 
relative electric field pulse parameters required for full electroporation of variable spheroid parameters. Electroporation of the model well beyond the threshold level maintained the primary results characteristics as indicated in Section 4 (data not shown).

\section{RESULTS}

To investigate the relative effects of close spheroid to spheroid proximity on electroporation behaviour, the electric field parameters required for threshold electroporation of isolated individual spheroids were first determined. Consideration of (1) indicates that the model induced transmembrane potential $U_{m}$, should be approximately directly proportional to $a$ for exponential decay pulses where $\tau_{p}$

$>\tau_{r}$, and much less dependent on $a$ for $\tau_{p}<<\tau_{r}$ as shown in previous modelling (Gaynor and Bodger 1995a). Using a membrane relative permittivity $\varepsilon_{r}$, of 3 (Gaynor and Bodger, 1994), membrane thickness $d$, of $30 \mu \mathrm{m}$ (Gaynor and Bodger, 1994), external media conductivity $\sigma_{e}$, of $6.0 \mathrm{mSm}^{-1}$, and internal media conductivity $\sigma_{i}$, of $14 \mathrm{mSm}^{-1}$, in (2) and (3), the $65 \mathrm{~mm}$ radius model has an approximate $\tau_{r}$ of $12 \mu \mathrm{s}$, whereas the $30 \mathrm{~mm}$ radius model has an approximate $\tau_{r}$ of $5.4 \mu \mathrm{s}$. Both of these time constants, while less than the $1 / e$ decay time constant, $\tau_{p}$, of the electric field pulses used, are within the same order of magnitude. Thus, it is expected that the induced transmembrane potential will be less than directly proportional to model radius, but still have considerable dependency on it. A direct proportionality of $U_{m}$ to model radius would result in a ratio of peak electric field amplitude required for threshold breakdown between the $65 \mathrm{~mm}$ radius model to the $30 \mathrm{~mm}$ radius model $\left(\mathrm{E}_{\mathrm{p}}(65\right.$ $\left.\mathrm{mm}): \mathrm{E}_{\mathrm{p}(30 \mathrm{~mm})}\right)$ of 2.17:1. The experimental results are shown in Table 1. There is an inverse dependency of threshold breakdown on model radius, where the smaller model requires a larger peak amplitude for breakdown. There is also an inverse relationship between the pulse decay time constant and peak amplitude, where shorter time constants require higher amplitudes. Both of these effects are well documented in biological electroporation (Neumann et al., 1989), and modelling (Gaynor and Bodger, 1995a). The results also show that the ratio between the electric field amplitude required for threshold breakdown in the large model to small model $\left(\mathrm{E}_{\mathrm{p}(65 \mathrm{~mm})}: \mathrm{E}_{\mathrm{p}(30 \mathrm{~mm})}\right)$ is reduced for the $10 \mu \mathrm{s}$ decay time constant pulse from $1.85: 1$ to $1.66: 1$, indicating a trend towards normalisation of 
electroporation for models of different sizes. This effect was also observed in the original balloon model system (Gaynor and Bodger, 1994, 1995b) and is a result of both the membrane relaxation time constant $\tau_{r}$, and the $U_{m}$ having a proportionality to the model radius $a$ as suggested by (1) and (2).

Threshold breakdown parameters for the three couplet structures are shown in Table 2. As expected, the couplets formed by two $65 \mathrm{~mm}$ radius models have the lowest electric field amplitudes required for breakdown. The couplets formed by two $30 \mathrm{~mm}$ radius models had the highest required electric field amplitudes. Finally, the couplets formed by one $65 \mathrm{~mm}$ and one $30 \mathrm{~mm}$ model had threshold breakdown electric field amplitudes marginally higher than the 65-65 couplet structure, and substantially lower than the 30-30 structure.

In all spheroid structures tested, the peak applied electric field amplitude required for threshold breakdown was lower than that required by equivalent size spheroids in isolation.

One effect of the reduced pulse decay time constant was an increase in applied electric field amplitudes required to induce membrane breakdown. The reduced pulse time constant also reduced the relative difference of the pulse amplitudes needed for electroporation, between all the structures.

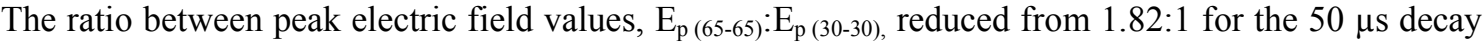
time constant pulse, to $1.60: 1$ for the $10 \mu$ s decay time constant pulse.

For all the couplet structures, threshold membrane breakdown only occurred in the immediate region of close couplet proximity. Super-threshold membrane breakdown arcing for a 65-30 couplet is shown in Figure 5 and a scanning electron microscope micrograph containing a typical electropore is presented in Figure 6. Droplets that formed on the dried surfaces of separated electroporated couplets generally showed a mirrored pattern, indicating that breakdown initially occurs at common points in the close proximity region between the two spheroids. 
Orientation of the couplets in the electroporation tank was critical for observed electroporation effects. If the close proximity region was rotated out of the normal axis to the electrode surface then no electroporation occurred at the lower threshold levels. Threshold electroporation for individual spheroids in the rotated couplets increased to their isolated values. This is also the result if couplets are not in very close proximity, forming a significant water-filled gap, even with no off-axis rotation. The gap size which increases threshold breakdown is relatively small (that is, approximately $1 \%$ of individual model radius).

Threshold breakdown for the basic-cubic $3 \times 3 \times 3$ array of $30 \mathrm{~mm}$ spheroids was the same as for the 30-30 couplets. The nine centre spheroids experienced breakdown in both contact regions lying on the electrode normal axis.

\section{DISCUSSION}

The fundamental finding of this study is that irrespective of pulse time constant values, compared to balloon models in isolation, the electric field amplitude required for threshold electroporation is substantially reduced for situations where close spheroid proximity exists in regions where the induced transmembrane potential is greatest, and the conductivity of the suspension media is a lower conductivity that the spheroid interior. Additionally, when small spheroids are close to large spheroids, electroporation can occur in the small model at electric field amplitudes close to the threshold value of close proximity between two large spheroids. The overall indicated result being, that a population of various spheroid sizes that are in close proximity can be close to uniformly electroporated with a pulse amplitude significantly lower than the threshold amplitude required for the large spheroid in isolation, given the spheroid external conductivity is less than its interior conductivity. This finding could be partially reflected by some in-vivo electroporation applications (Hofmann et al. 1999; Goto et al., 2000) that report optimum applied electric field amplitudes lower than generally required for similar cells electroporated in-vitro (Neumann et al., 1989), although this is difficult to accurately quantify owing to the range of pulse parameters that can occur across applications, especially the difference between external and cytoplasm conductivities. 
The reduction in pulse electric field amplitudes required to induce electroporation for the physical model spheroids in close proximity occurs primarily in response to the additional membrane compressive force induced by electro-deformation. Previous photographic evidence of membrane breakdown indicated that electro-deformation was present (Gaynor and Bodger 1994). Elongation of the arc points parallel to the applied electric field was observed, as expected for the experimental conditions where the external conductivity is lower than the spheroid interior. This elongation was much less evident in the multiple spheroid structures as the membranes in contact were more stationary owing to the membranes being forced together. While this electro-deformation compressive force has not been specifically calculated, supporting evidence from biological experimentation suggest that the force is significant enough to reduce the threshold for the onset of electropermeabilization. An additional factor that may also reduce the threshold for electroporation is the locally increased electric field amplitude created by the irregular-shaped, small-scale, discontinuous dielectric boundary between the spheroid and surrounding media (Qin, et al., 2005; Gowrishankar and Weaver, 2003; Khalifa, M., 1990). Small-scale membrane surface irregularities (see Figure 6) in the region of close proximity further increase the immediately local electric field amplitude which, when exceeding the threshold amplitude for electroporation, may result in joint membrane breakdown at those deformation points. Although solving (4) for very high volume fractions $\left(f_{0}>0.9\right)$, results in a slightly amplified transmembrane potential compared to cells in isolation, it is relatively much smaller than the reduction in electroporation threshold. The observed electro-deformation and electric field enhancement leading to threshold electroporation at reduced applied electric field strengths are not currently supported by any numerical models known to the authors.

The observation that reduction of electroporation threshold was not achieved when there was either a separation of the spheroids greater than approximately $1 \%$ of their diameter, or the close-proximity point was off-axis from the applied electric field, strongly supports the mechanism of electrodeformation, and to a lesser extent small-scale membrane deformation. Photographic evidence 
suggests that deformation is less than around 1\% of the spheroid diameter (Gaynor and Bodger, 1994). Therefore, any separation of a comparable magnitude will substantially reduce the effect of electrodeformation induced membrane compression, as the spheroids are free to deform into the separation region. Similarly, if the spheroid structures are off-axis, the elongation axis is not in-line with the close proximity point so the membranes will not experience electro-deformation compression. Separation will also not allow small-scale membrane surface irregularity-enhanced electric fields to affect the neighbouring membrane, as the enhanced field strength extends not much further than the scale of the deformation. Off-axis orientation reduces the overall, and hence local, electric field magnitude in the close proximity region by a factor of $\cos \phi$, to levels below that required for initiation of electroporation.

The observed electroporation sensitivity to orientation relative to the applied electric field suggests that for applications where there are regularly oriented, close cell to cell proximity structures (such as in muscle tissue for example), ensuring that the applied electric field orientation creates maximum membrane polarisation where the cells are in close proximity may enhance uniform electroporation and reduce required pulse amplitudes.

To further normalize the degree of electroporation for biological applications where there are multiple cell sizes, shapes and/or close contact, the model results suggest that it may be advantageous to use higher amplitude, shorter time constant pulses. This may be of concern for gene-transfer applications where it is sometimes considered that long pulse widths enhance DNA transfer. However, it has been shown that subsequent to the initial electroporation pulse, multiple lower-amplitude pulses can be used to maintain membrane permeabilization and enhance transfer (Satkauskas et al., 2002). Although it could be possible to non-uniformly electroporate an entire population of various cell sizes and shapes with longer time constant pulses that generate adequate permeabilization in the smaller cell sizes, this is likely to result in significant lysis of the larger cells undergoing extensive electropermeabilization (Weaver and Chizmadzhev, 1996; Meldrum et al., 1999; Smith et al. 2004). 
Electroporating cells in regions of close contact is often an initiator of cell to cell fusion (Neil and Zimmermann, 1993). It is not clear whether significant electrofusion is experienced with current invivo applications where the cells are in close proximity or even close contact, and has not yet been specifically investigated. There is some suggestion that electrofusion is not commonly observed for in-vivo applications (Zimmermann et al., 2000) where the external conductivity is greater than the cell cytoplasm such that cellular elongation deformation along the electric field axis is not experienced. For possible in-vivo applications where the external conductivity is lower than the cell cytoplasm, electrofusion could be initiated.

While the mechanism of membrane breakdown in the model is physically different to a biological cell, a number of the overall electrical characteristics are similar. The dielectric breakdown in the membrane of the model creates a low-impedance path for current flow through the induced pores. The resulting increase in membrane conductance is also a characteristic of biological electroporation, where the induced electropores contain both extracellular and intracellular media of a typically much higher conductivity than the lipid membrane. Additionally, with higher amplitude and longer duration pulses, the model pores increase in density and size which is again similarly characterized in biological electroporation. Importantly, Electro-deformation elongation parallel to the applied electric field is observed in both the model and biological cells when the suspending medium has a lower conductivity than the spheroid or cell interior. For multiple-pulse biological electroporation applications where the pulse repetition rate is much faster than the long-lived pore resealing rate, the model membrane has similar characteristics where its pores are filled with conducting media which influence subsequent pulse effects on the membrane. At this stage, the model is incapable of reproducing biological cell pore resealing dynamic characteristics.

\section{CONCLUSIONS}

By creating balloon model spheroid structures with close proximities, nonlinear effects on electroporation dynamics as a result of close proximity were observed. Whilst not an exact large scale 
analogy of biological cells, the electrical response of the model and cells to applied electric fields are fundamentally similar.

Applications of electroporation that involve environments where there is close cell to cell proximity and the exterior conductivity is lower than the cell cytoplast conductivity, need to be aware that there is a likely enhanced permeabilization effect due to the cellular environment. To maximize the uniformity of electroporation in these applications, pulse parameters should be accordingly adjusted and electric field orientation should be considered.

Future development of the model is required to determine the effect of increasing the external conductivity to levels higher than the spheroid interior to determine if the reduced threshold of electroporation is lost, as would be indicated by electro-deformation theory. Also, increasing the number of spheroids in the model structures beyond $3 \times 3 \times 3$ is required to provide a better representation of actual tissue where cell cluster numbers are very large, which has a bearing on the average electric field seen by cells deep within the cluster.

\section{ACKNOWLEDGEMENTS}

Substantial technical support for this work was provided by Jac Woudberg, Department of Electrical and Computer Engineering, University of Canterbury, New Zealand.

\section{REFERENCES}

Brandisky, K., and Daskalov, I. (1999): 'Electrical field and current distributions in electrochemotherapy', Bioelectrochemistry and Bioenergetics, 48, pp. 201-208.

De Bruin, K., and Krassowska, W. (1999): 'Modelling electroporation in a single cell. I. Effects of field strength and rest potential', Biophysical Journal, 77, pp 1213-1224. 
Gaynor, P. T. and Bodger, P. S., (1995a) 'Electrofusion processes: theoretical evaluation of high electric field effects on cellular transmembrane potentials', IEE Proceedings on Science, Measurement and Technology, Vol.142, No.2, pp.176-182.

Gaynor, P. T. and Bodger, P. S. (1995b): 'Balloon model of biological cell electropermeabilization in relation to the radius dependence of membrane dielectric breakdown', IEE Proceedings on Science Measurement and Technology, 142, pp. 277-282.

Gaynor, P. T. and Bodger, P. S. (1994): 'Ionisation of dielectric spheroid membranes: a balloon model of electroporation of biological cells', IEE Proceedings on Science Measurement and Technology, 141, pp. 190-196.

Gaynor, P. T., Wells, D. N., and Oback, B. (2005): 'Couplet alignment and improved electrofusion by dielectrophoresis for a zona-free high-throughput cloned embryo production system', Medical \& Biological Engineering \& Computing, 43, pp. 150-154.

Goto, T., Nishi, T., Tamura, T., Dev, S. B., Takeshima, H., Kochi, M., Yoshizato, K., Kuratsu, J., Sakata, T., Hofmann, G. A., and Ushio, Y. (2000): 'Highly efficient electro-gene therapy of solid tumor by using an expression plasmid for the herpes simplex virus thymidine kinase gene', Proc. Nat. Acad. Sci. USA, 97, pp. 354-359.

Gowrishankar, T. R., and Weaver, J. C. (2003): 'An approach to electrical modeling of single and multiple cells', Proc. Nat. Acad. Sci. USA, 100, pp. 3203-3208.

Hofmann, G. A., Dev, S. B., and Nanda, G. S. (1999): 'Electroporation therapy: A new approach for the treatment of head and neck cancer', IEEE Transactions on Biomedical Engineering, 46, pp. 752759.

Holzapfel, C., Vienken, J., and Zimmermann, U. (1982): 'Rotation of cells in an electric field', Journal of Membrane Biology, 67, pp. 13-26. 
Jayaram, S.H., and Boggs, S.A. (2004): 'Optimization of electroporation waveforms for cell sterilization', IEEE Transactions on Industry Applications, 40, pp. 1489-1497.

Jones, T. (1995): ‘Electromechanics of Particles’, Cambridge University Press, USA.

Khalifa, M. (ed.) (1990): 'High voltage engineering: Theory and practice’, Marcel Dekker Inc.

Kotnik, T., and Miklavčič, D. (2000): 'Analytical description of transmembrane voltage induced by electric fields on spheroidal cells', Biophysical Journal, 79, pp. 670-679.

Meldrum, R.A., Bowl, M., Bee-Ong, S., and Richardson, S. (1999): 'Optimisation of electroporation for biochemical experiments in live cells', Biochemical and Biophysical Research Communications, 256, pp. 235-239.

Misra, A., Ganga, S., and Upadhyay, P. (2000): 'Needle-free, non-adjuvanted skin immunization by electroporation-enhanced transdermal delivery of diphtheria toxoid and a candidate peptide vaccine against hepatitis B virus', Vaccine, 18, pp. 517-523.

Neil, G. and Zimmermann, U. (1993): ‘Electrofusion', Methods in Enzymology, 220, pp. 174-196.

Neumann, E., Kakorin, S., and Tœnsing, K. (1999): 'Fundamentals of electroporative delivery of drugs and genes', Bioelectrochemistry and Bioenergetics, 48, pp. 3-16.

Neumann, E., Sowers, A., and Jordan, C. (eds), (1989): 'Electroporation and Electrofusion in Cell Biology', Plennum Press, New York. 
Oback, B., Wiersma, A., Gaynor, P., Laible, G., Tucker, F., Oliver, J., Miller, A., Troskie, H., Wilson, K., Forsyth, J., Berg, M., Cockrem, K., McMillan, V., Tervit, H., and Wells, D. (2003): 'Cloned cattle derived from a novel zona-free in vitro production system', Cloning and Stem Cells, 5, pp. 3-12.

Pavlin, M., Pavšelj, N. and Miklavčič, D. (2002): 'Dependence of induced transmembrane potential on cell density, arrangement and cell position inside a cell system', IEEE Transactions on Biomedical Engineering, 49, pp. 605-612.

Qin, Y., Lai, S., Jiang, Y., Yang, T., and Wang, J. (2005): 'Transmembrane voltage induced on a cell membrane in suspensions exposed to an alternating field: A theoretical analysis', Bioelectrochemistry, 67, pp. 57-65.

Ramos, A., Raizer, A., and Marques, J. (2003): 'A new computational approach for electrical analysis of biological tissues', Bioelectrochemistry, 59, pp. 73-84.

Riske, K., and Dimova, R. (2005): 'Electro-deformation and poration of giant vesicles viewed with high temporal resolution', Biophysical Journal, 88, pp. 1143-1155.

Satkauskas, S., Bureau, M. F., Puc, M., Mahfoudi, A., Scherman, D., Miklavčič, D., and Mir, L. M. (2002): 'Mechanisms of in vivo DNA electrotransfer: Respective contributions of cell electropermeabilization and DNA electrophoresis', Molecular Therapy, 5, pp. 133-140.

Serša, G., Kranjc, S., and Čemažar, M. (2000): 'Improvement of combined modality therapy with cisplatin and radiation using electroporation of tumors', Int. J. Radiation Oncology Biol. Phys., 46, pp. 1037-1041.

Singh, B. N. and Dwivedi, C. (1999): 'Antitumor drug delivery by tissue electroporation', Anti-Cancer Drugs, 10, pp. 139-146. 
Smith, K., Neu, J., and Krassowska, W. (2004): 'Model of creation and evolution of stable electropores for DNA delivery', Biophysical Journal, 86, pp. 2813-2826.

Sukhorukov, V., Mussauer, H., and Zimmermann, U. (1998): 'The effect of electrical deformation forces on the electropermeabilization of erythrocyte membranes in low- and high-conductivity media', Journal of Membrane Biology, 163, pp. 235-245.

Tarek, M. (2005): ‘Membrane Electroporation: A Molecular DynamicsSimulation’ Biophysical Journal, 88, pp. 4045-4053.

Tatham, B.G., Giliam, K.J., and Trounson, A.O. (1996): 'Electrofusion parameters for nuclear transfer predicted using isofusion contours produced with bovine embryonic cells'. Mol. Reprod. Dev., 43, 306-312.

Weaver, J. C., and Chizmadzhev, Y. A. (1996): 'Theory of electroporation: A review', Bioelectrochemistry and Bioenergetics, 41, pp. 135-160.

Yamazaki, Y., Yagi, T., Ozaki, T., and Imoto, K. 2000 "In vivo gene transfer to mouse spermatogenic cells using green fluorescent protein as a marker”, Journal of Experimental Zoology, 286, pp. 212-218.

Zewert, T. E., Pliquett, U. F., Vanbever, R., Langer, R., and Weaver, J. C. (1999): 'Creation of transdermal pathways for macromolecule transport by skin electroporation and a low toxicity, pathway-enlarging molecule', Bioelectrochemistry and Bioenergetics, 49, pp. 11-20.

Zimmermann, U., Friedrich, U., Mussauer, H., Gessner, P., Hämel, K., and Sukhorukov, V. (2000): 'Electromanipulation of mammalian cells: Fundamentals and application', IEEE Transactions on Plasma Science, 28, pp. 72-82. 


\section{TABLES}

Table 1. Threshold electroporation pulse parameters for isolated spheroid balloon models.

\begin{tabular}{ccc}
\hline $\begin{array}{l}\text { Model Radius, } a \\
(\mathrm{~mm})\end{array}$ & $\begin{array}{l}\text { 1/e Decay Time } \\
\text { Constant }(\mu \mathrm{s})\end{array}$ & $\begin{array}{l}\text { Pulse Peak Amplitude, } \mathrm{E}_{\mathrm{p}} \\
\left(\mathrm{kV}_{\mathrm{p}} \mathrm{m}^{-1}\right)\end{array}$ \\
\hline $65 \pm 4$ & $50 \pm 2$ & $234 \pm 10$ \\
$65 \pm 4$ & $10 \pm 2$ & $290 \pm 10$ \\
$30 \pm 2$ & $50 \pm 2$ & $434 \pm 10$ \\
$30 \pm 2$ & $10 \pm 2$ & $482 \pm 10$ \\
\hline
\end{tabular}

Table 2. Threshold electroporation pulse parameters for spheroid couplet balloon model structures.

\begin{tabular}{ccc}
\hline $\begin{array}{c}\text { Couplet Structure } \\
\text { (radius-radius) }\end{array}$ & $\begin{array}{l}\text { 1/e Decay Time } \\
\text { Constant }(\mu \mathrm{s})\end{array}$ & $\begin{array}{l}\text { Pulse Peak Amplitude, } \mathrm{E}_{\mathrm{p}} \\
\left(\mathrm{kV}_{\mathrm{p}} \mathrm{m}^{-1}\right)\end{array}$ \\
\hline $65-65$ & $50 \pm 2$ & $152 \pm 10$ \\
$65-65$ & $10 \pm 2$ & $193 \pm 10$ \\
$30-30$ & $50 \pm 2$ & $276 \pm 10$ \\
$30-30$ & $10 \pm 2$ & $309 \pm 10$ \\
$65-30$ & $50 \pm 2$ & $180 \pm 10$ \\
$65-30$ & $10 \pm 2$ & $203 \pm 10$ \\
\hline
\end{tabular}




\section{LIST OF FIGURES}

Figure 1. Typical transmembrane potential magnitude distribution and electric field geometry around a primarily non-conducting membrane spheroid relative to a linear applied electric field.

Figure 2. Balloon model structure illustrations showing typical placement in the electroporation tank. (a) 65-65 couplet, (b) 65-30 couplet, (c) 30-30 couplet, and (d) $3 \times 3 \times 3$ array of $30 \mathrm{~mm}$ spheroids.

Figure 3. An oscilloscope trace of a typical $65 \mathrm{kVp}, 50 \mu \mathrm{s} 1 / \mathrm{e}$ decay time constant pulse. The time scale is $10 \mu \mathrm{s} / \mathrm{div}$, and the voltage scale is $20 \mathrm{kV} / \mathrm{div}$.

Figure 4. An illustration of the complete experimental setup (not drawn to scale).

Figure 5. (a) Typical placement of a 65-30 couplet in the electroporation tank, with (b) corresponding substantial membrane breakdown (electroporation) arcing in the region of close spheroid to spheroid

proximity $\left(E_{p}=200 \mathrm{kVm}^{-1}\right)$. Note there is no observable breakdown occurring at the opposite poles of the spheroids.

Figure 6. Oblique angle scanning electron microscope micrographs of (a) a typical section of a balloon model latex rubber membrane (note the irregular surface with local deformities that increases local electric field amplitude), and (b) a characteristic close proximity balloon model electropore. 


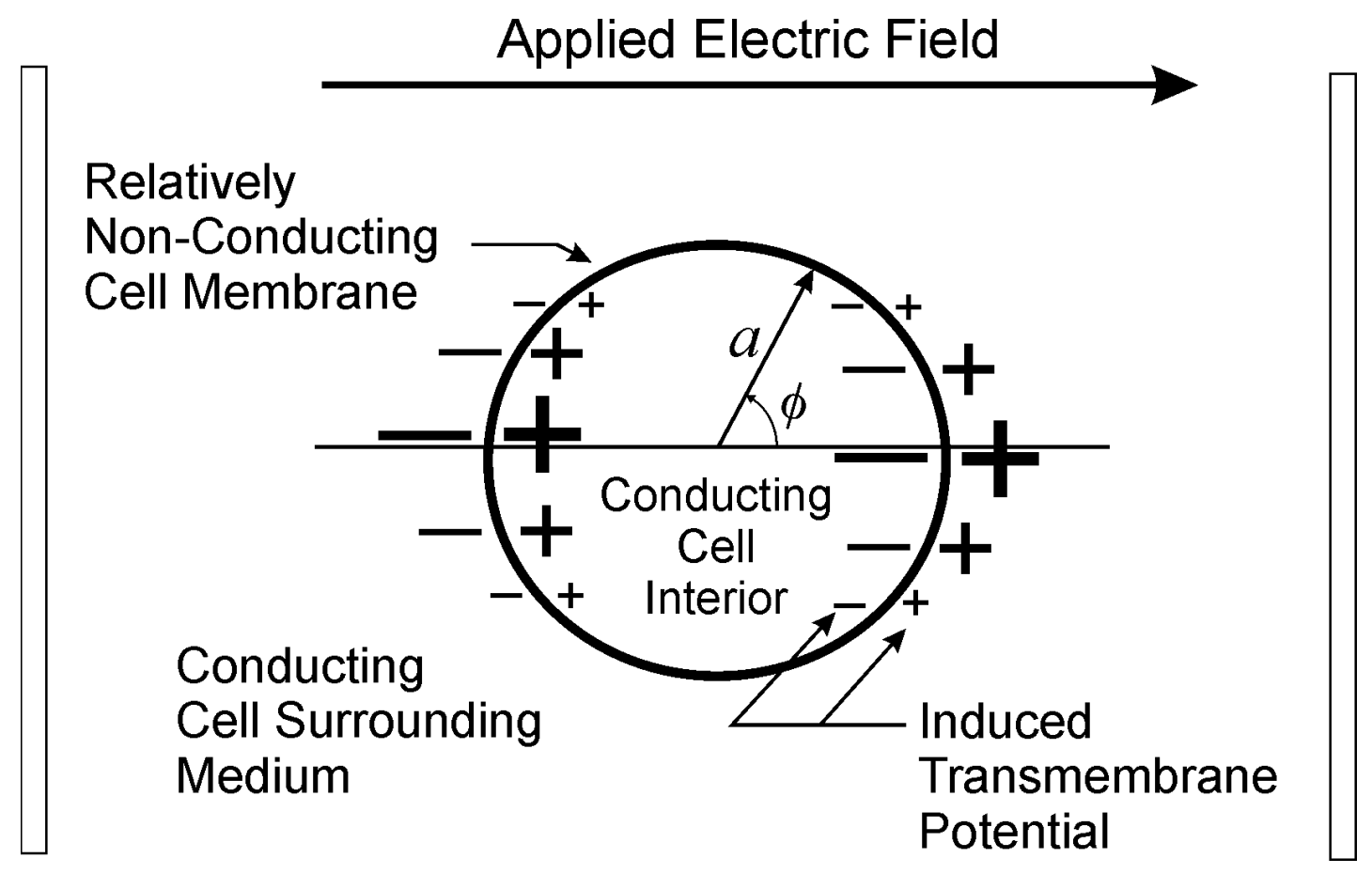

Figure 1. 


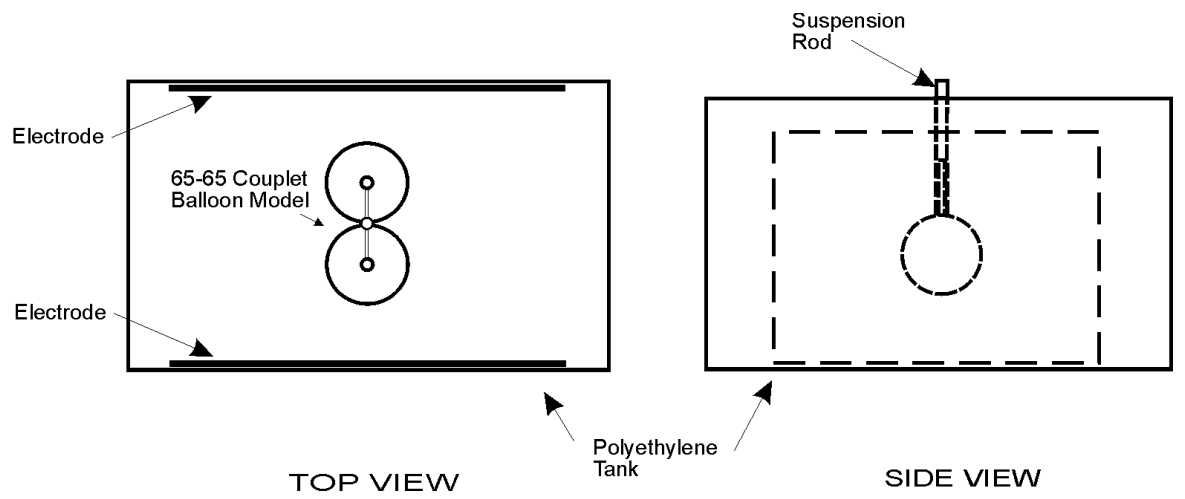

(a)

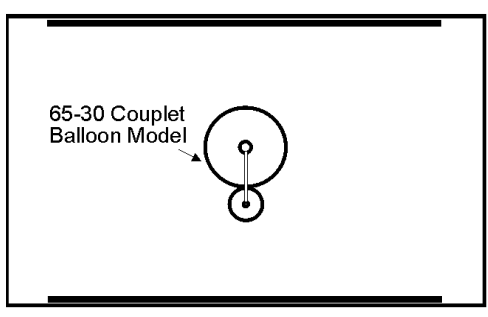

TOP VIEW

(b)

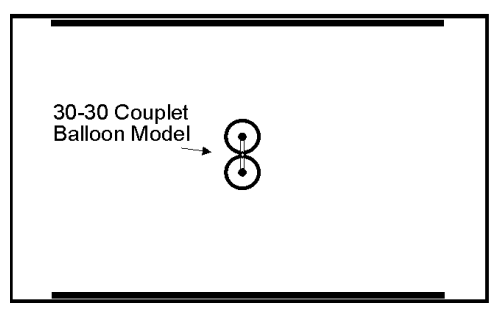

TOP VIEW

(c)

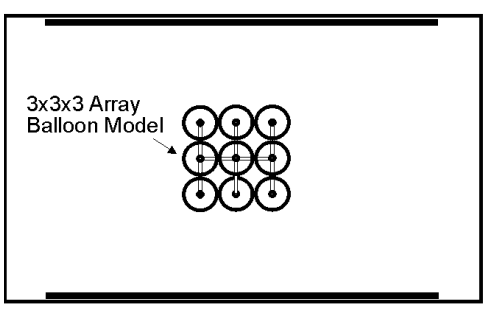

TOP VIEW

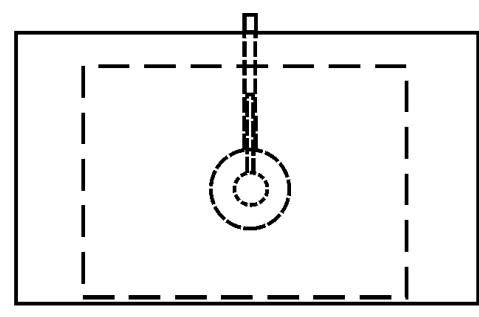

SIDE VIEW

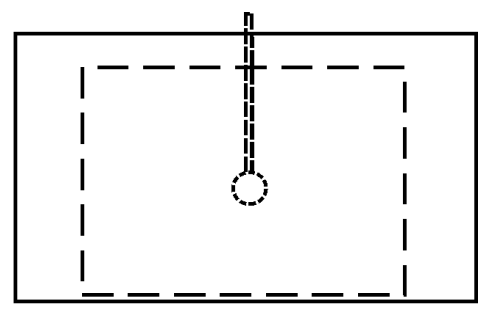

SIDE VIEW

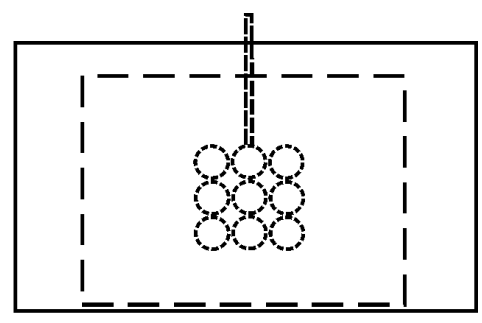

SIDE VIEW

(d)

Figure 2. 


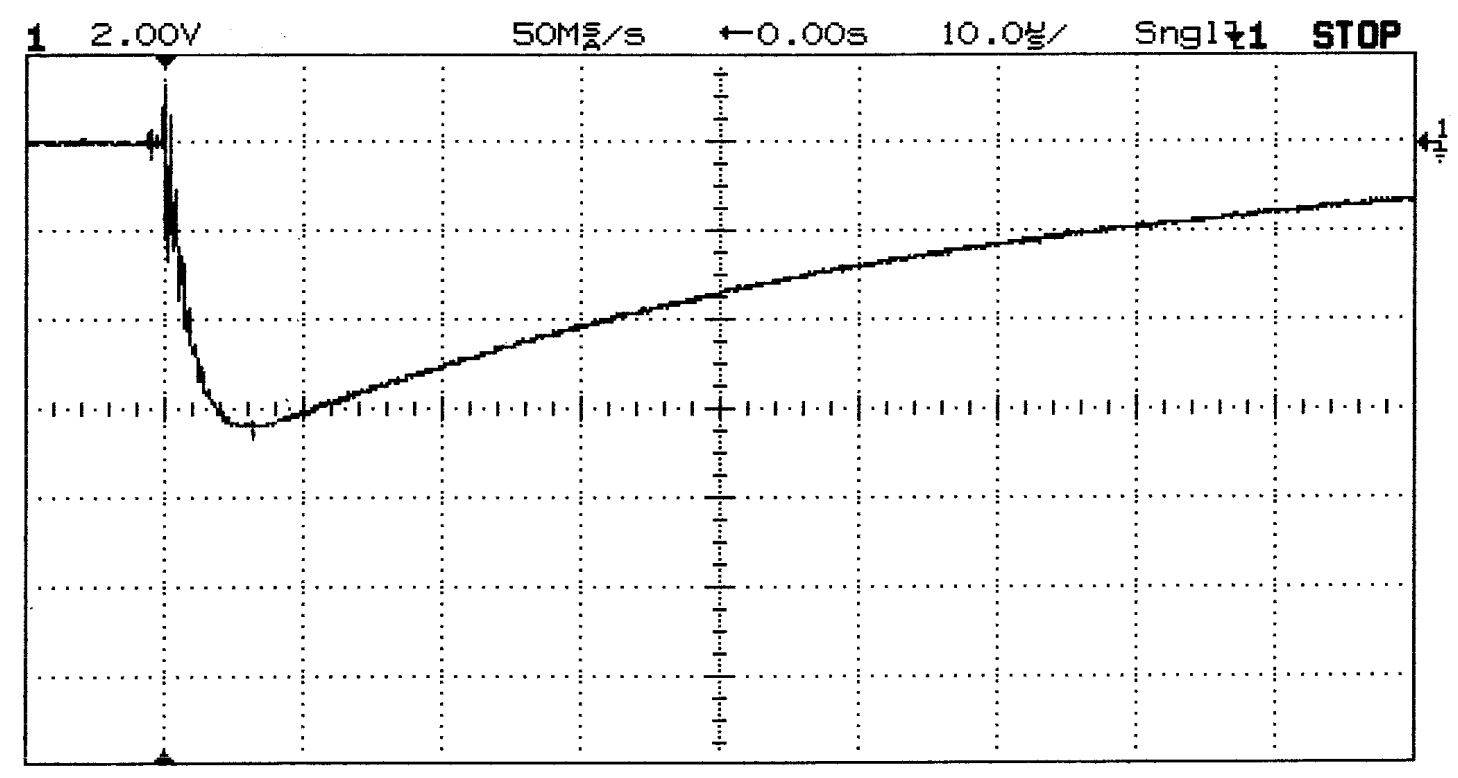

Figure 3. 

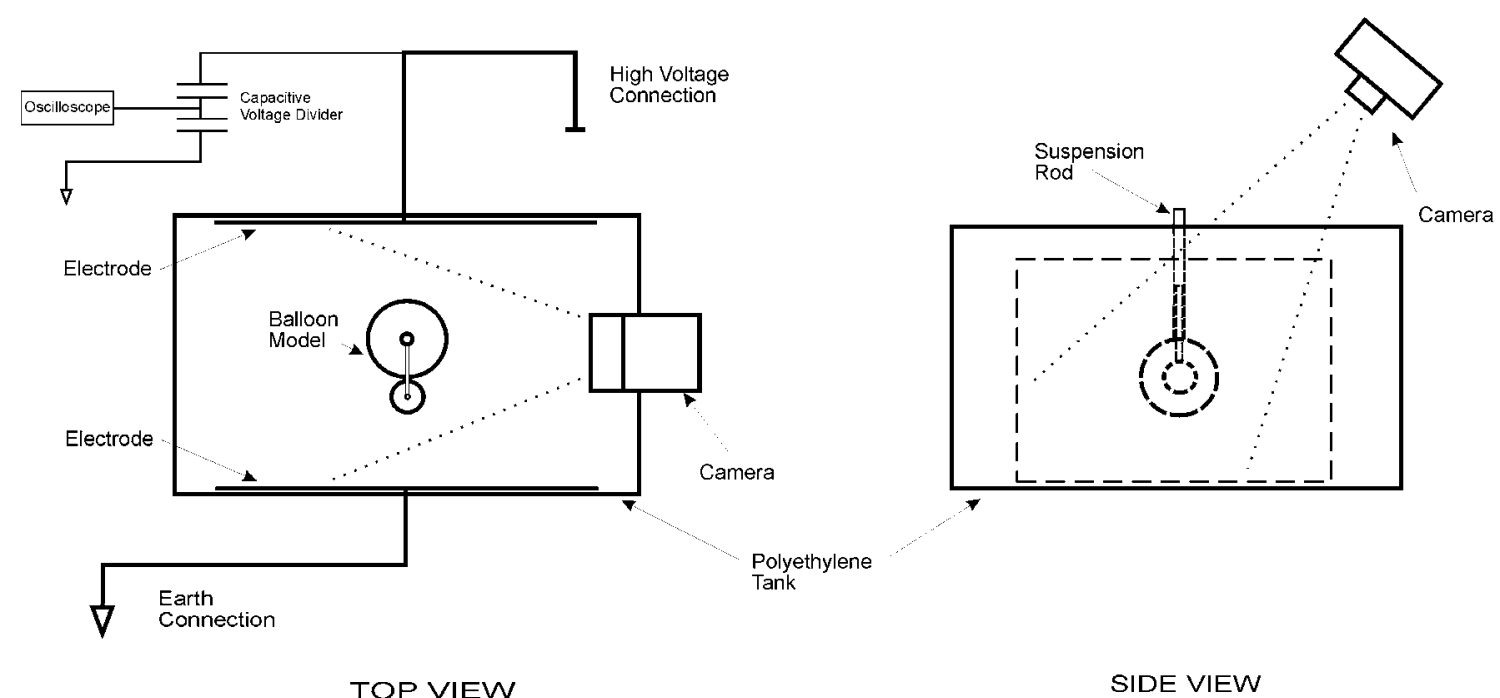

Figure 4. 


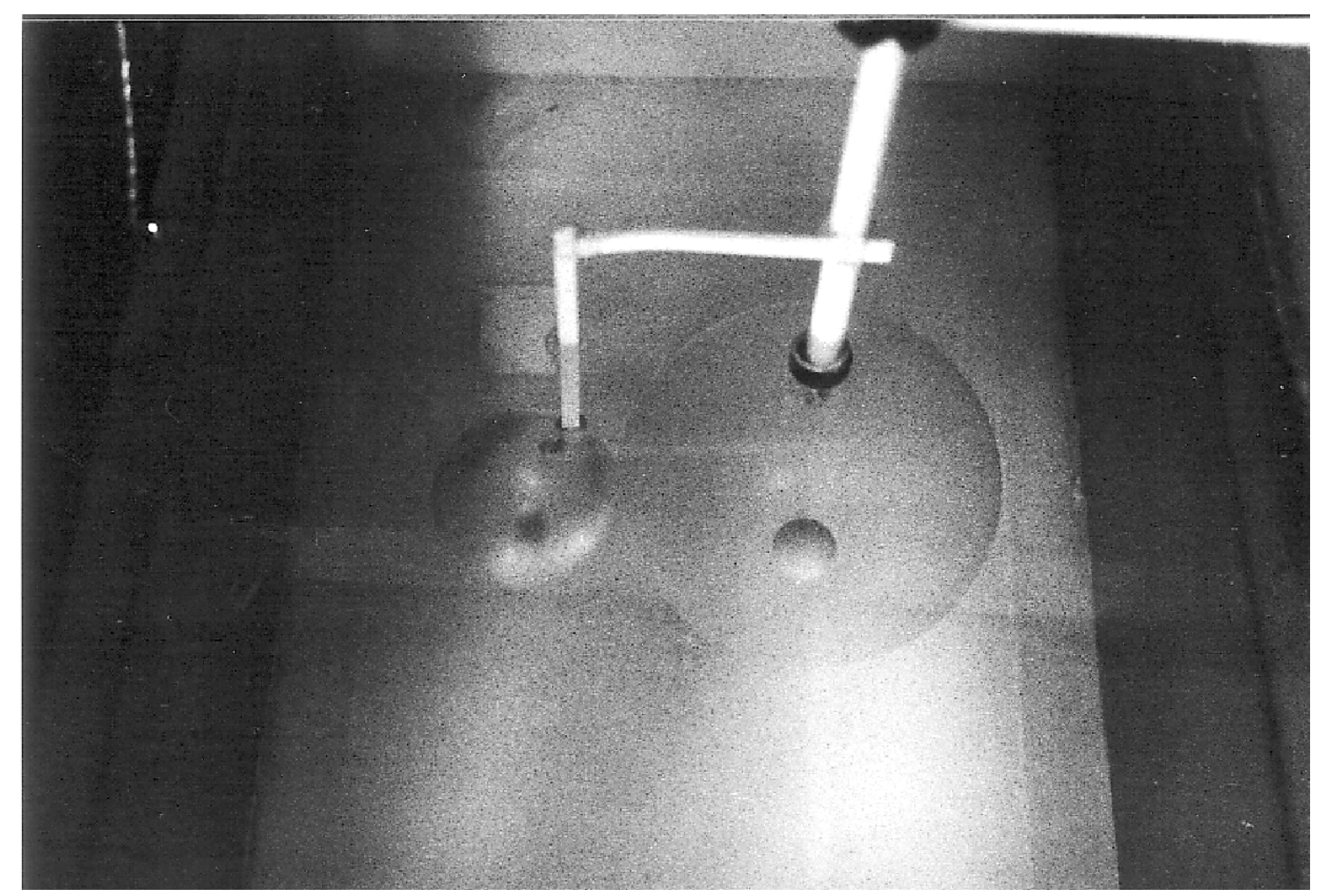

(a)

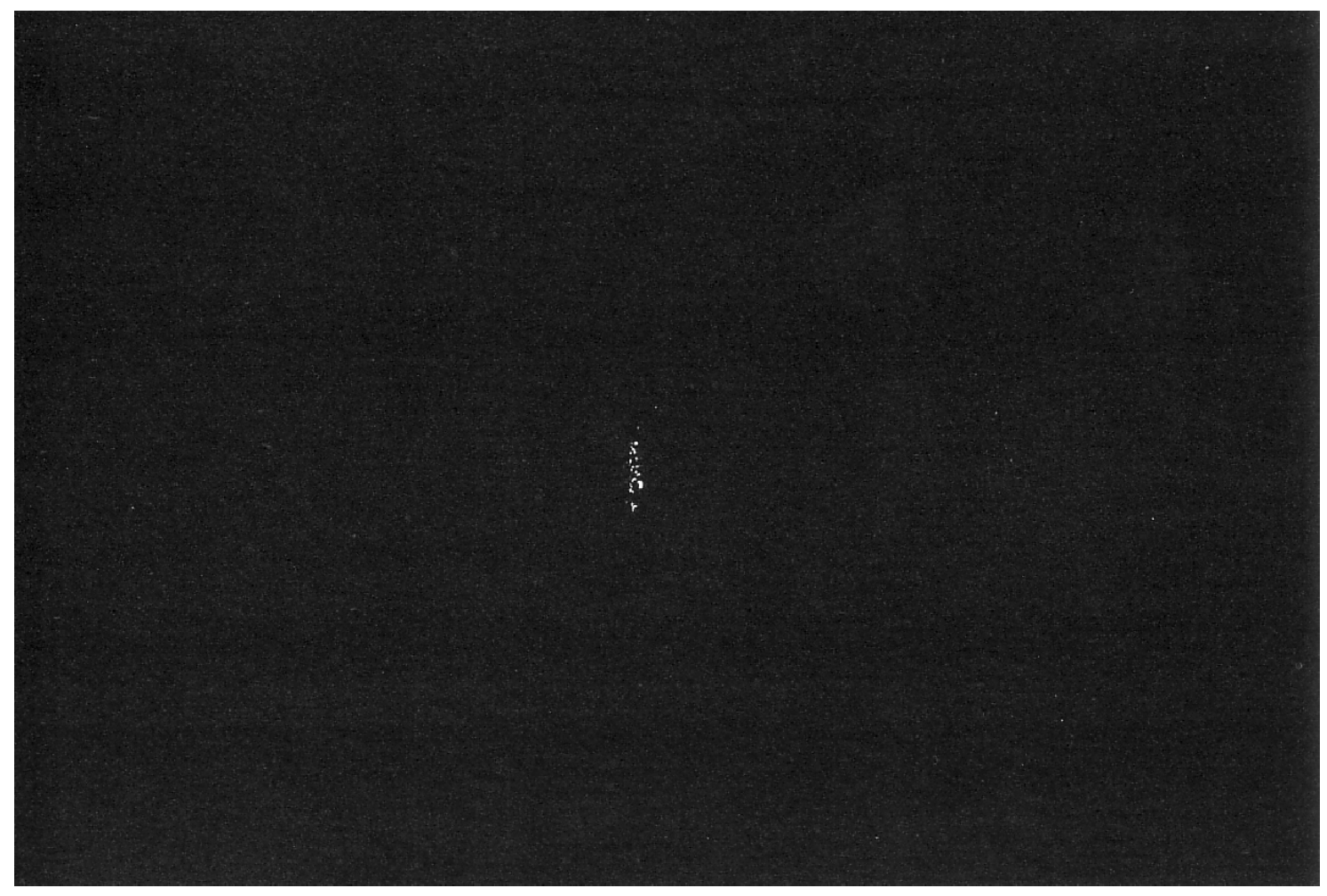

(b)

Figure 5. 


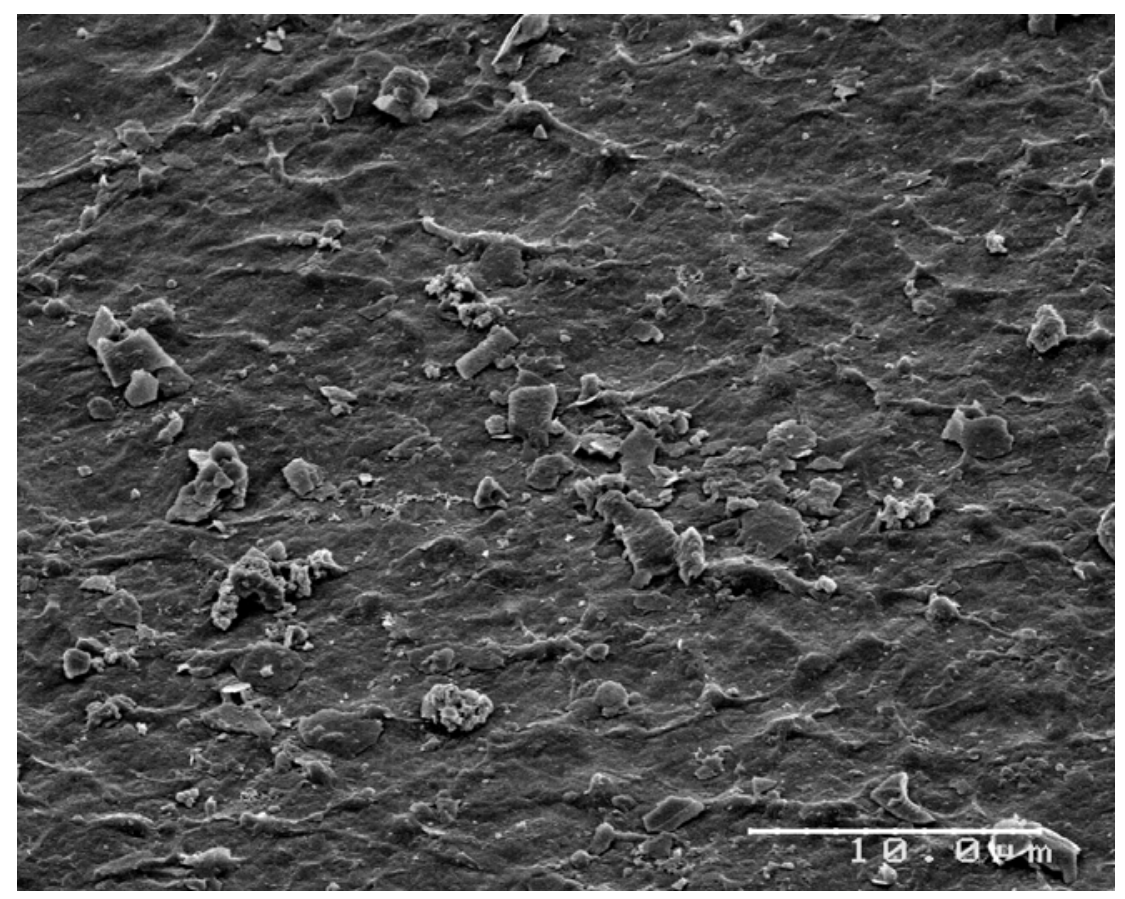

(a)

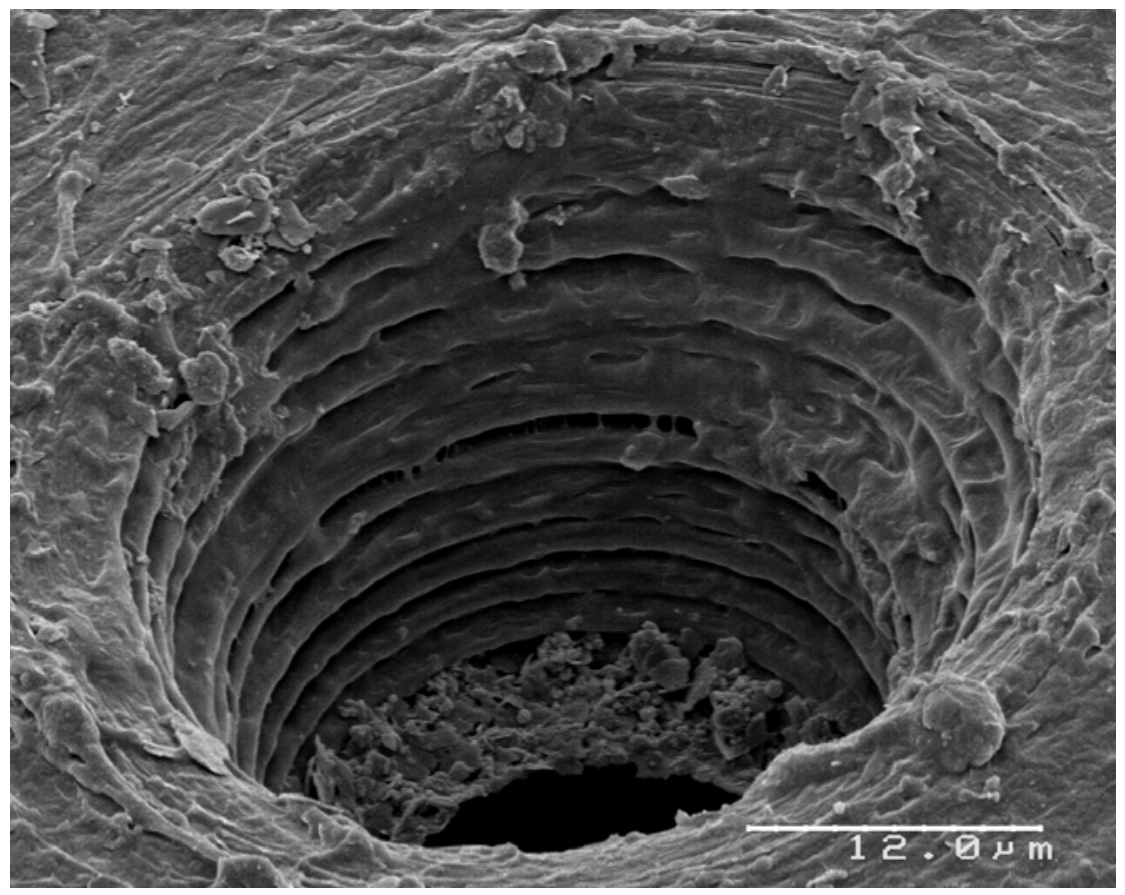

(b)

Figure 6. 